from: Prof. MA Gonzalez-Gay received grants/research supports from Abbvie, MSD, Jansen and Roche., Speakers bureau: Consultation fees/participation in company sponsored speaker's bureau from Pfizer, Lilly, Sobi, Celgene, Novartis, Roche and Sanofi., Ricardo Blanco Grant/research support from: Abbvie, MSD, and Roche, Consultant for: Abbvie, Pfizer Roche, Bristol-Myers, Janssen, Speakers bureau: Abbvie, Pfizer, Roche, Bristol-Myers, Janssen

DOI: 10.1136/annrheumdis-2019-eular.3621

\section{AB1099 CHAGAS DISEASE REACTIVATION IN THE RHEUMATOLOGIC IMMUNOSUPPRESSED PATIENT. IS IT NO LONGER AN ORPHAN DISEASE?}

Ariana Ringer $^{1}$, Juan Pablo Ruffino ${ }^{1}$, Nadia Cuadranti ${ }^{1}$, Florencia Martinez ${ }^{1}$, Cecilia Argento ${ }^{1}$, Ignacio Rolla ${ }^{1}$, Juan Manuel Vandale ${ }^{1}$, Carla Achilli ${ }^{1}$, Mariano Palatnik ${ }^{1}$, Noel Cortese ${ }^{1}$, Mariana Lagrutta ${ }^{2}$, Rodolfo Leiva ${ }^{3}$, Damian Aguila ${ }^{4}$, Laura Cordoba ${ }^{5}$, Milagros Zafra ${ }^{5}$, T Gambande $^{5}$, Patricia Sciarratta ${ }^{6}$, M Svetaz $^{6}$, F Gonzalez $^{7}$, F Pacini $^{7}$, Silvina Villar ${ }^{7}$, Ana Rosa Perez ${ }^{7}$, Oscar Bottasso ${ }^{7}$, Marcelo Abdala ${ }^{1}$. ${ }^{1}$ Reumatología, Hospital Provincial del Centenario, Universidad Nacional de Rosario, Rosario, Argentina; ${ }^{2}$ Clínica Médica, Hospital Provincial del Centenario, Rosario, Argentina; ${ }^{3}$ Cardiología, Hospital Provincial del Centenario, Rosario, Argentina; ${ }^{4}$ Infectología, Hospital Provincial del Centenario, Rosario, Argentina; ${ }^{5}$ Microbiología, Universidad Nacional de Rosario, Rosario, Argentina; ${ }^{6}$ Laboratorio Central, Facultad de Ciencias Bioquímicas y Farmaceúticas, UNR, Rosario, Argentina; ${ }^{7}$ Instituto de Inmunología Clínica y Experimental de Rosario (IDICER, CONICET, UNR), Rosario, Argentina

Background: Chagas' disease (CD) is an endemic and neglected infection in Latin America. Due to international human migration, it has become a worldwide issue. Scarce evidence is published regarding its behavior in rheumatic diseases (RD) with rheumatologic treatments (RT).

Objectives: To screen and follow up patients with CD under RT. To detect clinical and serological reactivation.

Methods: A systematic screening was conducted between January 2018 and January 2019 in a third-level Hospital in Argentina. Patients with CD and concomitant RD under RT were included. Assessments were done before and after RT. A direct (Strout) and indirect method (Polymerase chain reaction-PCR) were performed in order to detect parasitemia, levels of antibodies were evaluated by three techniques. Clinical, infectological and cardiological features were examined. Everything was assessed in, at least, two opportunities, separated by a minimum of one month. If treatment was modified or clinical condition changed, all the evaluations were repeated as before. In case of reactivation, specific treatment was indicated and follow up controls were stricter.

Results: 38 patients were identified. RD: Rheumatoid Arthritis: 22 $(57,9 \%)$, Systemic lupus erythematosus: $4(10,5 \%)$, Systemic Sclerosis: 3 (7,9\%), Vasculitis: 2 (5,3\%), Psoriatic Arthritis: 1 (2,6\%), others: 6 $(15,8 \%)$. RT: classic, synthetic and biological disease-modifying antirheumatic drugs, cyclophosphamide and corticosteroids.

Two reactivations were detected with both direct and indirect methods, with a significant title antibody increment and consistent clinical signs and symptoms. Cardiological abnormalities were found. A 64-years old lady with Microscopic Polyangiitis, under prednisone $60 \mathrm{mg} /$ day and a 57-years old lady with Systemic Lupus Erythematosus under hydroxychloroquine and prednisone $40 \mathrm{mg} / \mathrm{day}$, who happen to be sisters. They did not have other treatments. Both of them developed high fever, myalgias, arthralgias and asthenia. Other infections were ruled out. They received Benznidazole and Nifurtimox respectively. After one-week treatment, Strout and PCR became negative and antibodies decreased, with remarkable clinical improvement.

Conclusion: Two patients with parasitemia and concomitant symptoms were detected, after high doses of corticosteroids. Because they were sisters, a genetic background would also play a role. Parasite's screening before starting immunosuppression and its follow-up during treatment in search of reactivation should be integrated to rheumatology daily practice in endemic countries. It should also be taken into account in patients from non-endemic countries that have an epidemiological nexus. CD specific antibodies determination, Strout and PCR are economic and practical techniques, although they require qualified personnel and equipment. In areas with high prevalence of $C D$, the benefits of this measures would outweigh costs. A more advanced study should be performed to extend our knowledge.

Disclosure of Interests: None declared

DOI: 10.1136/annrheumdis-2019-eular.3509

\section{$\mathrm{AB} 1100$ \\ PREVALENCE OF SARCOPENIA IN ECUADORIAN POPULATION BASED ON SCREENING AND DIAGNOSTIC TOOLS}

Genessis Maldonado, Carlos Rios. Espiritu Santo University, Rheumatology, Guayaquil, Ecuador

Background: Sarcopenia is defined as an abnormal loss of muscle mass associated with aging. It has been validated that sarcopenia can predict functional deterioration.

Objectives: The purpose of this study is to determine the diagnosis of sarcopenia by conventional methods and gold standard.

Methods: Prospective observational study of patients attending a rheumatology center, to whom anthropometric and densitometric measurements were taken in order to determine sarcopenia.

Results: A total of 110 patients were studied, from which $91 \%$ were women and $9 \%$ men. $66 \%$ presented sarcopenia by means of the gold standard test (DXA). The mean age was 65.80 \pm 9.84 [44-89]. The mean age for women was $66.2 \pm 9.9$ [44-86] and the average age of menopause was $46.08 \pm 8.53$ and $63 \%$ were found to have sarcopenia. In men, the mean age was $73.3 \pm 13.8$ [63-89] and $30 \%$ had sarcopenia.

Anthropometric measures were determined. The mean body mass index was $26.58 \pm 4.67$, which corresponds to overweight. Three tests were performed in addition to the gold standard. The average gait speed test was $1.12 \pm 0.74$ seconds and $45 \%$ were positive for sarcopenia. The mean of the muscle strength test (dynamometer) was 19.33 9.52 and $63 \%$ had sarcopenia. The mean of the SARC-F screening questionnaire was $3.02 \pm 2.24$ and $22 \%$ were positive for sarcopenia. According to the measurement of muscle mass by DXA, the mean appendicular skeletal muscle mass (ASMM) was 16.08 +3.52 , ASMM index $6.77 \pm 1.12$ and the total skeletal muscle was 21.39 \pm 4.68 . Men presented a higher muscle mass index compared to women $[7.82 \pm 1.93$ vs. $6.66 \pm 0.95]$. When group ing patients by BMI, sarcopenia was present in all of the patients with low weight, $88 \%$ in the ideal weight group, $67 \%$ in the overweight group and $25 \%$ in the obesity group. A statistically significant relationship was found between sarcopenia and sex [0.01], weight and height $[<0.001]$ gait test [0.04], muscle strength test $[<0.001]$ and DXA $[<0.001]$. In addition, the sensitivity and specificity of the tests were determined. SARC-F had a $15 \%$ sensitivity and a $9 \%$ specificity, with a positive predictive value of $74 \%$ and a negative predictive value of $37 \%$. The gait speed test $24 \%$ sensitivity and $38 \%$ specificity, with a positive predictive value of $57 \%$ and negative predictive value of $27 \%$. The muscle strength test had a $78 \%$ sensitivity and $65 \%$ specificity, with a positive predictive value of $84 \%$ and negative predictive value of $96 \%$ (Figure 1).

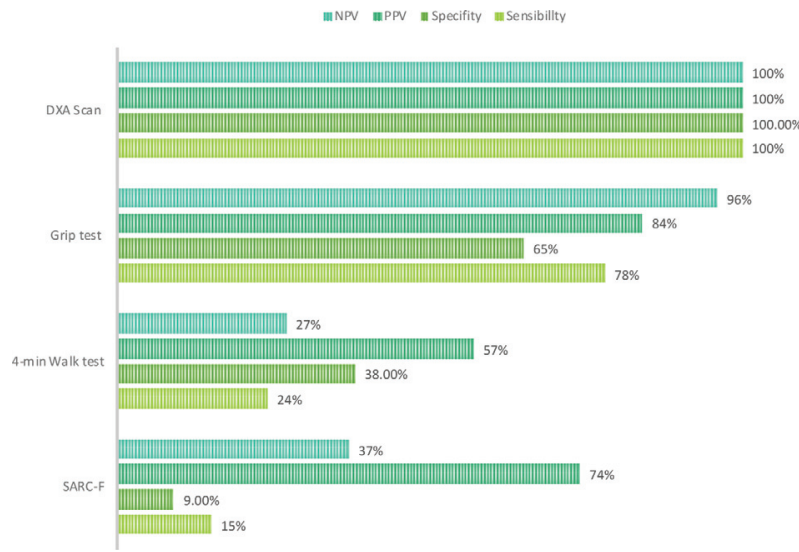

Abstract AB1100 Figure 1. Statistical measures of sarcopenia's diagnostic tools.

Conclusion: This is the first study of determination of Sarcopenia in a Rheumatology Center in the city of Guayaquil. $66 \%$ of the studied population presented sarcopenia. It is evident that conventional methods may determine a diagnosis, however, a definitive diagnosis can be established by DEXA. Because Sarcopenia is a multidisciplinary entity, more studies are needed to determine the usefulness of the conventional methods. Disclosure of Interests: None declared

DOI: 10.1136/annrheumdis-2019-eular.7374 